\title{
The Never-Ending Promise
}

Many thanks to Dr Gail Donner for her willingness to champion this Special Focus issue, but more importantly for her expertise and leadership in advancing the home and community care agenda in Ontario. To all of our contributors, your efforts are appreciated; thank you for your leadership, thoughtful reflections and call to action. The possibilities for a different "person-centred," "people-powered" home and community-oriented health system can be realized with nursing at the helm. But much like Gail, I am surprised and somewhat disappointed in the nursing community's underwhelming advocacy for an acceleration of the seemingly never-ending promise of shifting and transforming care delivery models. While there are some wonderful examples of innovative approaches to home and community care across the country - many led by nurses - why aren't they being more highly profiled for their positive outcomes, and advanced for cross-jurisdictional adoption by health ministries?

Beyond the issues identified by our contributors, we know there are many other related structure and process changes that also need to be addressed to achieve and sustain any new models. A transformed system of home and community care needs to be underpinned by certain functional elements including but not limited to: 1) shared and consistent approaches in the documentation of care - assessments, interventions, and outcomes, including those recorded by clients and families; 2) a electronic health record infrastructure that seamlessly affords access to critical information across care settings; 3) multiple options for accessing home and community care services when and where they are needed; 4) practical integration and "bundling" of health and social services as they relate to home-based care and support, and 5) access to an interprofessional team of resources which may or may not be led by nurses but ensures that clients and families have access to the expertise they need when they need it.

Together we are the community, we have a professional community - let's use it to our mutual benefit. It's time to deliver on the never-ending promise.

Lynn M. Nagle, RN, PhD, FAAN

Assistant Professor

Lawrence S. Bloomberg Faculty of Nursing

University of Toronto

Toronto, ON 